\title{
ENERGY CONSUMPTION REDUCTION IN WIRELESS SENSOR NETWORK BASED ON CLUSTERING
}

\author{
Gholamreza Farahani \\ Department of Electrical Engineering and Information Technology, Iranian Research \\ Organization for Science and Technology (IROST), Tehran, Iran
}

\begin{abstract}
One of the important issues in the routing protocol design in Wireless Sensor Networks (WSNs) is minimizing energy consumption and maximizing network lift time. Nowadays networks and information systems are one of the main parts of modern life that without them, people cannot live. On the hand, the impairment of these networks leads to great and incalculable costs. In this paper, a new method based on clustering has presented that problem of energy consumption is solved. The proposed algorithm is that energy-based clustering can create clusters of the same energy level and distribute energy efficiency across the WNS nodes. This proposed clustering protocol classify network nodes based on energy and neighbourhood criteria and attempts to better balance energy in clusters and ultimately increase network lifetime and maintain network coverage. Results are shown that the proposed algorithm is on average $40 \%$ better than LEACH algorithm and 14\% better than IBLEACH algorithm.
\end{abstract}

\section{KEYWORDS}

Wireless Sensor Network, Clustering, LEACH Algorithm, IBLEACH Algorithm

\section{INTRODUCTION}

WSNs are a special type of computer networks that have found wide applications due to its high capacity. Flexibility, self-organization, low-cost and rapid deployment are key features of ideal WSNs for many applications such as data gathering, military environment, environment monitoring, intelligent control, traffic management, medical operations and etc. These networks are composed of many small sensor nodes with high capability, low energy consumption and cheap price that are able to sense the parameters such as humidity, temperature, pressure and etc. from surrounding environment and send them to the sink node.

Despite improvements in these networks, because of the large number and small size of sensor nodes, they require batteries with low power. Also due to the usage of these networks in the harsh and unavailable environment, it is not possible to recharge or change the sensor nodes. Therefore, one of the important problems in the WSN is the energy limitation problem [1].

Ideal WSN should consume little energy and has an intelligent planning. It should be able to receive data quickly and accurately over the long time. Also its installation cost should be cheap and it does not require maintenance. During recent years, sensor networks have been attended significantly, especially with creation and extension of Micro Electro Mechanical Systems (MEMS) technology that makes it easy to build intelligent sensors [2]. These sensors are small and their mathematical and computational resources are limited that in comparison with traditional sensors are cheaper. 
Intelligent sensor nodes are low power devices that include one or multiple sensors, a processor, memory, energy source, radio (transmitter and receiver) and actuator. Different types of sensors such as mechanical, temperature, environmental, chemical, optical and magnetic sensors may be added to the sensor node to measure desired features of the environment. Since sensor nodes have limited memory and are usually distributed in environments where access to them is difficult, radio transmitters for wireless communications to send information and data to the base station (for example, a mobile computer, a personal computer or a point of connection to a fixed infrastructure) are considered [3].

The battery is the primary energy source in a sensor node and replacing or recharging the sensor battery is almost impossible due to the lack of continuous access to the sensors or their numerous numbers of them. The secondary energy source is the energy that gains a sensor node from the environment, which can be referenced to solar panels that may be added to the nodes, depending on the environment where the sensor is deployed [4].

By equipping the intelligent network with wireless sensors, much of the cost of data transfer is reduced; the network configuration becomes more organized and thus parallel processing and flexibility in these networks increases. As mentioned, the two directions of the smart grid are one of the important features of the network, in which customers will report the amount of energy they generate and their amount of energy to the grid. This connection can be defined within a country. In WSNs, where information is exchanged bilaterally, it also has the ability to monitor, repair and maintain in real time. A WSN can include several hundred to thousands of sensor nodes, each node capable of recording and transmitting physical or environmental changes. Therefore, it can be used in a variety of projects, including wind or solar power plants [5]. WSN is a kind of technology that is used today in three main areas: production, distribution and power consumption in smart electrical networks [6]. WSNs are one of the most important tools for gaining information and understanding of the environment that wide researches are focused on it. In general, WSNs can be classified from two aspects [7]. a) Sensing desired parameters from the environment, b) connection establishment for sending information to the sink node.

Generally, these networks have fix nodes or nodes with the very limited movement that all nodes send their information directly (single step) or indirectly (multiple steps) toward the sink node [8]. Usually WSN includes a central station and gateway that can connect to the number of sensors via radio link. Data will collect via these wireless sensors and will send to the gateway. Then the transmitted data by the gateway connection will use [9]. The control and monitoring centre collects information about the energy consumption of sensor nodes in a period or the amount of energy produced in a given area, and therefore provides appropriate instructions for increasing or reducing power generation.

The continuation of this paper, section 2 introduces clustering in WSN, section 3 presents the proposed algorithm, section 4 provides the simulation and section 5 concludes the paper.

\section{Clustering in WSN}

In cluster-based routing methods, nodes with more energy can be used to process and send information, while fewer energy nodes can be used to sense in the vicinity of the target. In fact, the hierarchy process by creating clusters and assigning specific tasks to cluster heads can have a major contribution to the scalability, lifetime, and overall energy efficiency of the system and avoid single-pass architecture. 
Hierarchical routing by performing the data collection and combination will reduce the number of messages sent to the base station which is an efficient way to consume less energy in a cluster. Hierarchical routing is mainly a two-layer routing, with one layer used to select cluster headers and another layer for routing. However, most of the technologies in this category are not about routing, but more of them are about who and when to send or process the information, the channel assignment, and etc. Clustering routing methods are potentially the most effective methods for reducing energy consumption in sensor networks and they have used a lot of applications in recent years [10]. The most prominent protocol of this category is Low Energy Adaptive Clustering Hierarchy (LEACH) algorithm [11]. This protocol inspired the development of many other clustering protocols, that each of them attempted to resolve some of its problems. An extension of LEACH algorithm which balances the energy consumption in the network is named intra-balanced LEACH (IBLEACH) [12]. This protocol has a better network lifetime and lowers energy consumption in comparison with LEACH algorithm. Due to the inherent characteristics of WSNs, the energy-aware routing problem has great importance. The most effective and most useful routing methods are cluster-based methods. Optimal clustering of nodes and the precise determination of the cluster head can play an effective role in energy-aware clustering algorithms and significantly increase the lifetime of WSNs [13].

A single level network may cause overhead in the head of clusters as the density of sensors increases. This overhead may result delay in communication and inadequate follow-up of occurrences. In addition, a single level architecture for a large set of nodes that covers a wide area is not scalable because usually, the sensors do not have the ability to communicate in a long way. Hierarchical clustering in WSNs can significantly affect the global scalability of the system, life time, energy efficiency and delay. Hierarchical routing is an efficient method for consuming less energy in a cluster, aggregating and combining data to reduce the number of messages sent to the base station, and most importantly, the significant drop in delay for transmitting data to the base station [14]. Scalability in this field requires load balancing and appropriate utilization of resources as well as applications requiring effective data aggregation.

Clustering, in addition to supporting network scalability and reducing energy consumption (through aggregation of data), has many other benefits as well as different goals. On the other hand, clustering can make the network topology stable at the sensor level and reduce the overhead and overall maintenance costs of the topology, meaning that the sensors are kept only when connected to their cluster heads, and changes in levels between cluster heads are not affected. Also, cluster head can implement optimized management strategies that enhance network performance and increase the battery life of the nodes, resulting in longer network lifetime.

A cluster head can schedule intra-clustering activities, which will allow nodes to switch to sleep mode and reduce energy consumption. Additionally, the nodes can be used in a Round-Robin sequence and specify a specific time for sending and receiving data; therefore, retransmission is prevented and the redundancy (data) in the covered area is reduced and avoid medium access collision [15]. Some important considerations in the process of designing a clustering algorithm for WSNs should be considered, including:

\section{A) Formation of clusters}

Cluster head selection procedures and cluster shaping should create the best possible clusters (for example, clusters are well balanced), however, they should be able to keep the number of exchange messages low and total time complexity should be, if possible, constant and independent of the growth of the network. 


\section{B) Application dependency}

When designing clustering and routing protocols for WSNs, application capability should be at high priority and designed protocols must have the ability to adapt to different types of desired application requirements.

C) Secure communication

Data security is important in WSN networks as much as it mattered in traditional networks [16]. The ability to design clustering of WSNs is more important to provide secure communication when these networks are considered for military applications.

D) Synchronization

Slot transfer schemes such as Time Division Multiple Access (TDMA) enable nodes to periodically schedule time gaps (in order to reduce energy consumption). In such plans, the mechanism of synchronization and the effect of this mechanism should be considered.

E) Data aggregation

Since this process has made it possible to optimize energy, it still remains one of the most fundamental challenges to design of the WSN. However, its effective implementation has not easy and straightforward procedures for many applications, and should be optimized to fit the requirements of the application.

\subsection{LEACH Algorithm}

The LEACH Network is made up of nodes, some of which are called cluster heads. The job of the cluster head is to collect data from their surrounding nodes and pass it on to the base station. LEACH is dynamic because the job of cluster head rotates. Cluster heads can be chosen stochastically (randomly based) on this algorithm as equation (1).

$$
T(n)= \begin{cases}\frac{P}{1-P \times\left(r \bmod P^{-1}\right)} & \forall n \in G \\ T(n)=0 & \forall n \notin G\end{cases}
$$

where $n$ is a random number between 0 and 1. $P$ is the desired percentage of cluster heads, $G$ is the set of nodes that were not cluster head in the last $P^{-1}$ rounds and $r$ is the current round. If $n<T(n)$, then that node becomes a cluster head. The algorithm is designed so that each node becomes a cluster head at least once [17].

\subsection{Parameters of Sensor Clustering}

Parameters are used as important tools for comparing and evaluating performance. In this section, cluster parameters are introduced which are as below:

\section{A) Number of clusters}

In most probabilistic clustering algorithms, cluster head selection and cluster formation process naturally leads to the creation of different clusters. However, in some published approaches, the 
set of cluster headings has been predefined; therefore, the number of clusters has been presented [18]. Usually, inefficiency of total routing protocol, the number of clusters is a key parameter.

B) Inter-cluster communication

In some of the early clustering approaches, the connection between a sensor and its head cluster is considered directly (single-step), while in newer protocols (according to network requirements), multiple interconnections are needed. Such as those in which the communication range of the sensor nodes is limited or the number of sensor nodes is too much and, consequently, the number of sub-clusters should be high.

C) The mobility of cluster heads and member nodes

If members or cluster heads are fixed, the management of inter cluster and intra clusters will be facilitated. In contrast, if nodes and cluster heads are considered as mobile, the membership in cluster for each group changes dynamically, and the boundary between the clusters changes in a moment and they are recruited instantaneously. Logically, they need more memory to store information.

D) Types of nodes and their role

In some heterogeneous network models, it is assumed that the cluster heads are more equipped with more communication and computing resources than other nodes. But in the homogeneous networks, all nodes have the same capabilities and are selected from the nodes of the cluster head members. In this paper assumed that network is homogeneous and cluster heads are selected from among the members.

\section{E) Cluster shaping structure}

In many recent approaches, when cluster heads are only typical sensor nodes (and the timing is one of the main design criteria), clustering will carry out as a distributed method and uncoordinated manner. But in some early approaches, a centralized approach (hybrid) has been followed; it means that one or more coordinating nodes are used to partition the entire network off-line and control membership in the cluster.

\section{F) Selection of cluster heads}

In many of the proposed algorithms, the groups of cluster head are preselected. While in many cases, cluster heads are selected from a distributed set of nodes, in probabilistic methods, either in a completely randomized manner or based on specific criteria (such as residual energy or connectivity) [19-21].

G) Complexity of the algorithm

At the end of each period, a series of data from the nodes must be transmitted to the base station. Therefore, the time complexity or convergence rate is an important argument in choosing an algorithm. According to the approach that is used, it can be fixed or variable. In some networks, it depends on the number of clusters or the total number of initial nodes.

H) Multiple levels

Using a hierarchical multiple levels clustering approach will result in less power consumption, less collision, and lower latency in data transfer. The use of multiple levels clustering, especially 
when faced with a large network, becomes more prominent, because in these networks the efficiency of the intra cluster communication is of great importance. On the other hand, multiple layering is one of the reasons for reducing the complexity of time and tasks are carried out simultaneously [22].

I) Overlapping

Some protocols have a particular attention to the concept of nodal overlap in different clusters (for better routing functionality, for faster execution of cluster shaping protocols or for other reasons). Many approaches try to minimize overlap or non-overlap (under no conditions). In a hierarchical clustering approach, there is no overlap on the defined network, and this is one of the other strengths of using this protocol.

\section{Proposed Algorithm}

In this section, a new clustering protocol is proposed. The incentive to create the proposed method is a neglecting of previous clustering algorithms to nodal energy levels, as the main parameter for network cluster formation. The proposed algorithm has been to improve the traditional idea of clustering (location clustering) to provide a common method for locationenergy clustering, in order to reach the main goal of WSNs, namely, increasing network lifetime while maintaining network coverage. The flowchart of figure 1 shows the main steps of the proposed method.

As shown in figure 1, routing in the proposed method has two basic steps as follows:

- Clustering

- Optimization of cluster centres by usage of energy criteria

In the following subsections, each of these steps will describe.

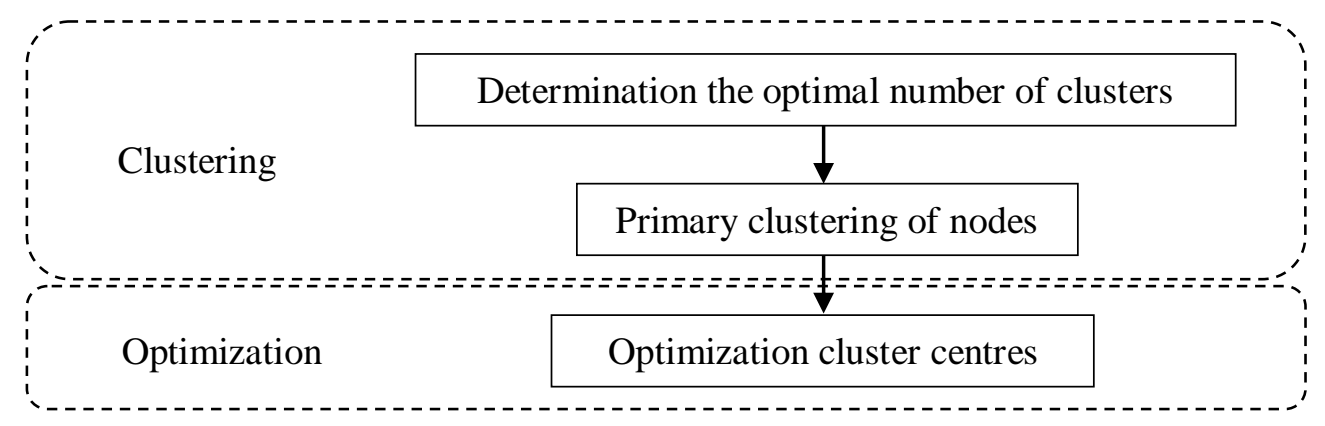

Figure 1. Flowchart of proposed method

\subsection{Clustering}

The main purpose of this stage of the proposed method is to divide sensor network nodes into the number of clusters. The clustering stage of the sensor nodes consists of two steps. In the first step, based on the validation indices, the optimal number of clusters will determine with the use of kmeans clustering algorithm. This algorithm depends on several factors, such as the number of clusters and the distance between the clusters. One of the most important issues in clustering is selecting the number of suitable clusters. In general, the number of clusters is proper if there are two following condition. 
- Existing samples in a cluster are as similar as possible. The common criterion for determining the data compactness is the variance of data.

- Samples belonging to different clusters should be separated as far as possible.

The above statements can also be expressed in this way that the clusters should have the maximum compression and, as far as possible, their separation is high. The disadvantage of all clustering algorithms is their separation from the cluster definition.

You can define clustering as follows: An unobservable sorting method that does not have any prior knowledge of the categories. In all existing methods, at least the default is determination the number of clusters to provide a suitable clustering solution. This contradicts the premise of unobservable classification in the definition of clustering. The k-means algorithm is one of the suitable methods for clustering, which requires the number of clusters to be specified at the beginning of the algorithm. The purpose of validating clusters is to find clusters that have the best fit with the desired data. The two base criteria for evaluating and selecting optimal clusters are [23]:

- Compactness: Data belonging to a cluster should be as close as possible to each other. A common criterion for determining the data compactness is the variance of data.

- Separation: The clusters themselves must be sufficiently separate from one another.

In general, there are three ways to measure and calculate the degree of cluster separation:

- Distance between the closest data from two clusters

- Distance between the farthest data from two clusters

- Distance between centres of clusters

One of the most popular indicators for determining the optimal number of clusters is the Root Mean Square Standard Deviation (RMSSDT). Although this criterion is usually used in the validation of hierarchical algorithms, it also has the ability to evaluate the results of other clustering techniques. In the RMSSDT, the variance of clusters is used, which can be calculated using equation (2).

$$
R M S S D T=\sqrt{\frac{\sum_{\substack{i=1 \ldots n_{F} \\ j=1 \ldots d}} \sum_{k=1}^{n_{k}}\left(x_{k}-\bar{X}_{j}\right)^{2}}{\sum_{\substack{i=1 . \ldots n_{F} \\ j=1 . . . d}}\left(n_{i j}-1\right)}}
$$

where $n_{i j}$ is the number of data in the $j$-th dimension of $i$-th cluster, $n_{F}$ is number of clusters, $d$ is the dimension of data, $n_{k}$ is number of data and $\bar{X}_{j}$ is the mathematical expectation of the $j$-th dimension of the data.

With respect to the equation (2), this criterion measures the degree of homogeneity of the clusters; therefore the smaller value of criterion means the better clustering of data. After determining the optimal number of clusters, sensor network nodes are split into a number of clusters. One of the most famous methods in this field is k-means algorithm, which despite the dependence on the initial conditions and the convergence to the local optimal points; these algorithms classify nk numbers of data into a $\mathrm{nF}$ cluster with high speed. 
K-means algorithm was presented by McQueen in 1967 [24] which categorizes data vectors in a D-dimensional space into the clusters that their numbers are already specified. Usually, the centre of the primary clusters is randomly selected from the original samples. Therefore, the clusters obtained in clustering are not unique because the centre of the primary clusters can be different in two independent clustering k-means. This category is based on the Euclidean distance between the data and the cluster centres, which are considered to be the similarity criterion. The Euclidean distance between the data vectors of a cluster with the centre of that cluster is less than their Euclidean distance with other cluster centres.

In general, the standard k-means method can be summarized in the following steps:

1. From the $n$ point of data, the $k$ point as the centre of the cluster named $m_{l}, m_{2}, m_{3} \ldots m_{k}$ will select randomly.

2. Each point of data will assign to its own cluster using the equation (3).

$$
S_{i}(t)=\left\{x_{p}:\left\|x_{p}-m_{i}(t)\right\|^{2} \leq\left\|x_{p}-m_{j}(t)\right\|^{2} \forall j, 1 \leq j \leq k\right\}
$$

where $S_{i}(t)$ represents the cluster associated with the data $i$ in the repetition $t$. 3. New cluster centres are calculated as follows:

$$
m_{i}(\mathrm{t}+1)=\frac{1}{\left|S_{i}(\mathrm{t})\right|} \sum_{x_{j} \in S_{i}(\mathrm{t})}^{\infty} x_{j}
$$

In simple and concise terms, the pseudo-code of the k-means algorithm can be expressed as follows:

$$
\begin{aligned}
& \text { Initialize } \mathbf{m}_{\mathrm{i}}, \mathrm{i}=1, \ldots, \mathrm{k} \text {, for example, to } \mathrm{k} \text { random } \mathbf{x}^{\mathrm{t}} \\
& \text { Repeat } \\
& \text { For all } \mathbf{x}^{\mathrm{t}} \text { in } \mathrm{X} \\
& \mathrm{b}_{\mathrm{i}}^{\mathrm{t}} \leftarrow 1 \text { if }\left\|\mathbf{x}^{\mathrm{t}}-\mathbf{m}_{\mathrm{i}}\right\|=\min _{\mathrm{j}}\left\|\mathbf{x}^{\mathrm{t}}-\mathbf{m}_{\mathrm{j}}\right\| \\
& \mathrm{b}_{\mathrm{i}}{ }^{\mathrm{t}} \leftarrow 0 \text { otherwise } \\
& \text { For all } \mathbf{m}_{\mathrm{i}}, \mathrm{i}=1, \ldots, \mathrm{k} \\
& \text { Until } \mathbf{m}_{\mathrm{i}} \text { converge } \\
& \mathbf{m}_{\mathrm{i}} \leftarrow \text { sum over } \mathrm{t}\left(\mathrm{b}_{\mathrm{i}}{ }^{\mathrm{t}} \mathbf{x}^{\mathrm{t}}\right) / \operatorname{sum} \text { over } \mathrm{t}\left(\mathrm{b}_{\mathrm{i}}{ }^{\mathrm{t}}\right)
\end{aligned}
$$

The goal of the clustering of the k-means algorithm is to determine the cluster centres to be selected in such a way as to minimize the intra cluster distance, as well as the clusters have a highest distance from each other.

\subsection{Optimization Cluster Centres}

The main objective of this step is to select a cluster centre from each cluster according to a series of defined criteria. To select cluster centres, the following criteria are used:

1. $f_{1}$ : Total energy of selected cluster centres

2. $f_{2}$ : Total distance of nodes of that cluster from the centre of the cluster

3. $f_{3}$ : The total distance of the selected cluster centres from each other 
If first criterion is higher and the two other criteria are lower, better cluster centres is carried out. As a result, the proposed method is a multi-objective algorithm that needs to be optimized.

In the proposed method, according to the multi-objective nature of the problem, the fitness function is defined as the optimal selection of cluster heads. The ultimate fitness function is defined as follow:

$$
\text { fitness }=\alpha f_{1}+\beta \frac{1}{f_{2}}+\gamma \frac{1}{f_{3}}
$$

where $\alpha, \beta$ and $\gamma$ are constant parameters that determine the effect of three different criteria. The purpose at equation (5) is maximizing the objective function. Since the second and third criteria must be minimized, therefore they are inversely proportional to the fitness function.

\section{SimULATION}

This section is intended to simulate the proposed methodology presented in the previous section. In the beginning, the appropriate tool has been explained and then the network assumptions have been addressed, then the proposed method has been evaluated in three different situations and the results of these three conditions have been shown. System specifications for implementing the proposed method include $2.5 \mathrm{GHz}$ processor, 4GB physical RAM, Windows 10 operating system, and MATLAB 2017b software implementation tool.

\subsection{Network assumptions}

The network assumptions are as follows:

A) Sensor nodes are randomly located.

B) All sensor nodes and base station are fixed after the deployment stage.

C) The nodes are able to adjust the transmit power according to the distance from the receiver node.

D) Distance between nodes can be calculated based on received signal strength. Therefore, the exact location of the sensor nodes is not required.

E) All sensor nodes have the same energy at the beginning of the deployment.

\subsection{Assessment of proposed method}

To compare the proposed method with LEACH and IBLEACH methods, due to position of the station, the number of 100, 200 and 300 nodes is considered. Then, separately, for 100, 200 and 300 nodes, the number of live nodes, the energy remaining in different periods, the First Node Dies (FND) and Half of the Node Dies (HND) values, and the number of cluster heads in the three different methods are evaluated. In simulation, the parameters $\alpha, \beta$ and $\gamma$ are $0.7,0.3$ and 0.2 respectively. The size of the data packet is 4000 bit, primary energy of each node is 10 Joule, $E_{\text {elec }}$ and $\mathrm{E}_{\mathrm{amp}}$ are energy consumption in Electronic and amplifier transmission circuit respectively and their values is considered 0.05 and 0.1 Joule respectively. The probability of being cluster head is considered as $5 \%$. 


\subsubsection{Results for 100 nodes}

The network configuration for 100 nodes along with initial 5 clusters and their cluster heads with our proposed method are shown in figure 2 . The network size is $100 \times 100$.

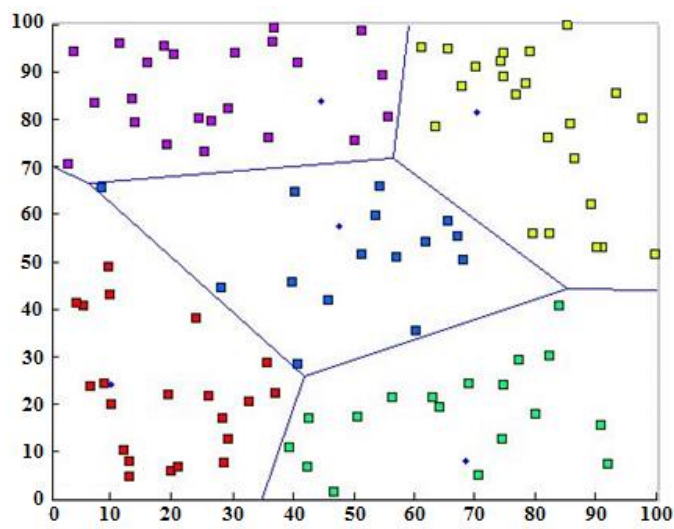

Figure 2. Network configuration for 100 nodes, 5 clusters and their cluster heads of proposed method with network size of $100 \times 100$

Table 1 shows the simulation results in round 500. As shown in table 1, the proposed algorithm has a better performance than LEACH and IBLEACH in terms of FND and HND.

Table 1. FND and HND values of network size $100 \times 100$ in round 500

\begin{tabular}{|l|l|l|}
\hline Method & HND & FND \\
\hline LEACH & 675 & 345 \\
\hline IBLEACH & 785 & 350 \\
\hline Proposed Method & 885 & 435 \\
\hline
\end{tabular}

In this case, LEACH has the weakest performance among the three algorithms. Figure 3 shows the FND and HND values in this case. Using this graph and the values in table 1 it is clear that if the FND criterion is considered, the proposed method is $26 \%$ better than LEACH and $24 \%$ better than IBLEACH, and if the HND criterion is used, the proposed method is $31 \%$ better than LEACH and about $13 \%$ better than IBLEACH.

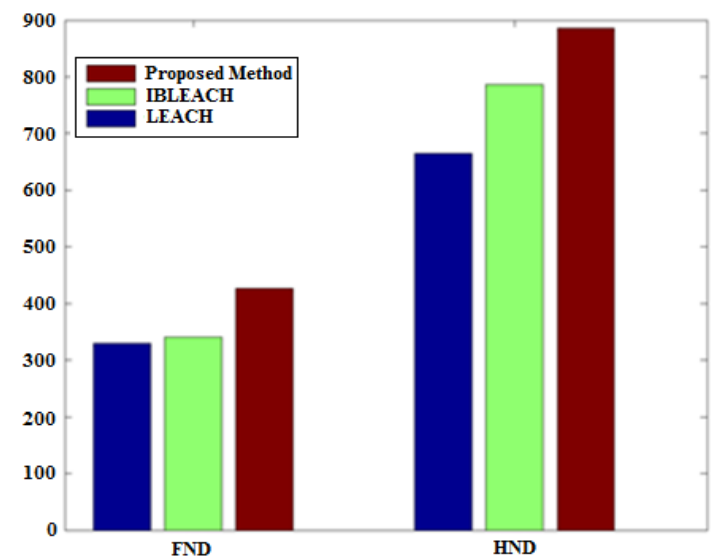

Figure 3. Comparison the FND and HND of the proposed method with IBLEACH and LEACH algorithms for 100 nodes in round 500 
Because LEACH does not consider the residual energy level of the sensor nodes during clustering, it has the weakest performance. This algorithm uses a pure probabilistic model for clustering that is not enough to obtain the best solution. Since IBLEACH considers energy parameters and local distances in each cluster cycle, it has a better performance than LEACH. The IBLEACH method and the proposed method consider the level of energy of any cluster head to compute the clustering radius. This means that if the cluster head has more energy, it will have a larger cluster radius. In other words, there are more sensor nodes in that location. This feature ensures more work is assigned to the cluster head that has more energy. This idea makes our proposed algorithm to perform better than other algorithms in this case.

Figure 4 illustrates the number of live sensor nodes according to the number of rounds for each algorithm. This figure clearly shows that the mortality of sensor nodes in the proposed method begins after all other algorithms. As shown in figure 5, the energy efficiency of proposed algorithm is compared with other algorithms. Since each sensor node has 10 Joule initial energy, the total energy of the wireless sensor network is initially 1000 Joule. The battery of each node is discharged by increasing each round.

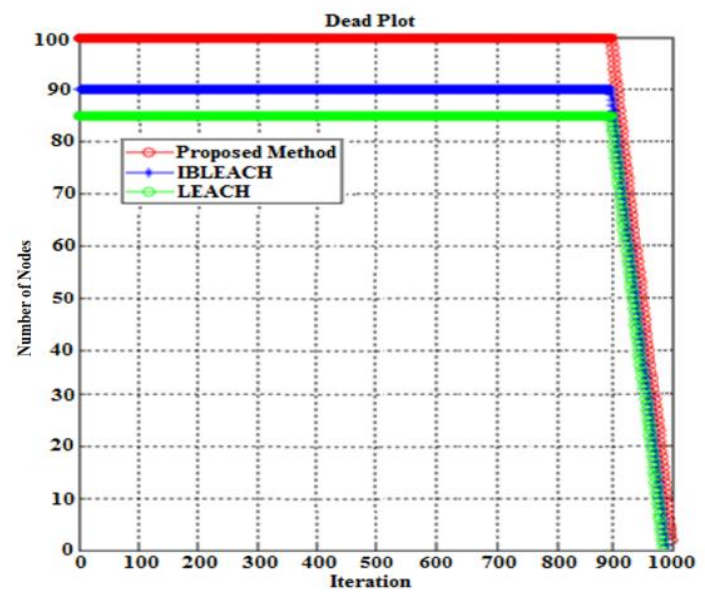

Figure 4. Number of network live nodes in 100 nodes for proposed method, IBLEACH and LEACH algorithms in 1000 rounds

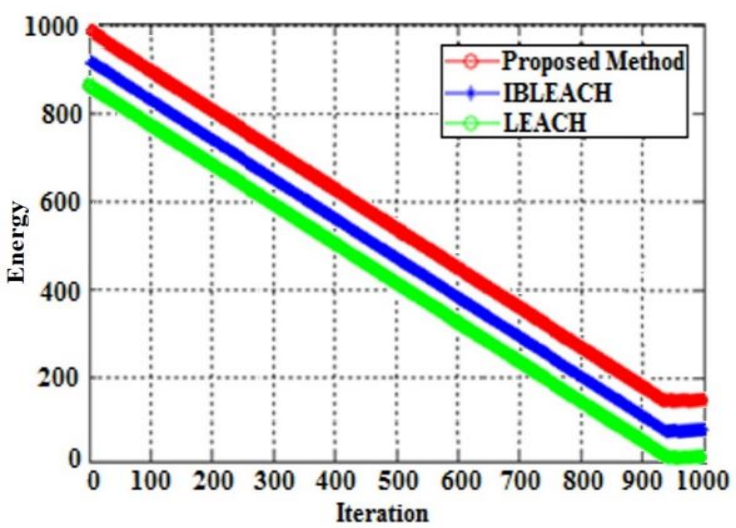

Figure 5. The remaining energy at 100 nodes for proposed method, IBLEACH and LEACH algorithms in 1000 rounds 
At Round 1000, LEACH has the lowest energy level of about 15 Jules. The energy level of IBLEACH is approximately equal to 82 Jules. On the other hand, the proposed method has the highest energy level of 154 Jules. This result is concluded alongside the FND and HND criteria, indicating that our proposed method is $927 \%$ higher than LEACH and about $88 \%$ higher than IBLEACH.

The number of cluster heads in different methods versus rounds for 100 nodes is shown in Figure 6. In this case, because the number of clusters decreases by decreasing the energy of nodes, the chart is descending. On the other hand, since the selection of cluster head is probable, the shape of the graph is zigzagically goes high and low.

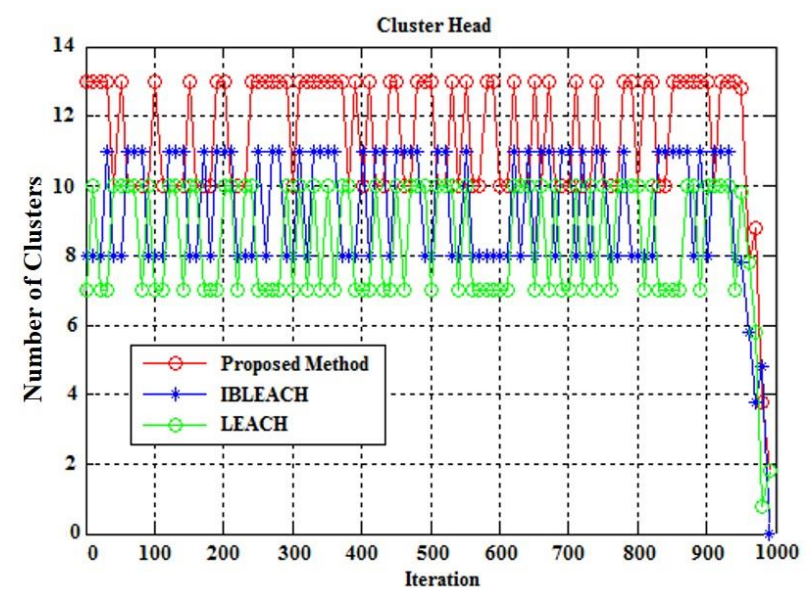

Figure 6. Number of cluster heads versus rounds for 100 nodes in 1000 rounds

\subsubsection{Results for 200 nodes}

In this case, as in the first case, the initial clusters are 5 and the network size is $200 \times 200$ (figure 7). In this case, the density of the sensor nodes deployment is twice of the previous case. The purpose of this case is to test the behaviour of algorithms in the topology of different sensor networks with a different number of sensor nodes. The environment and assessment conditions are presented in table 2 .

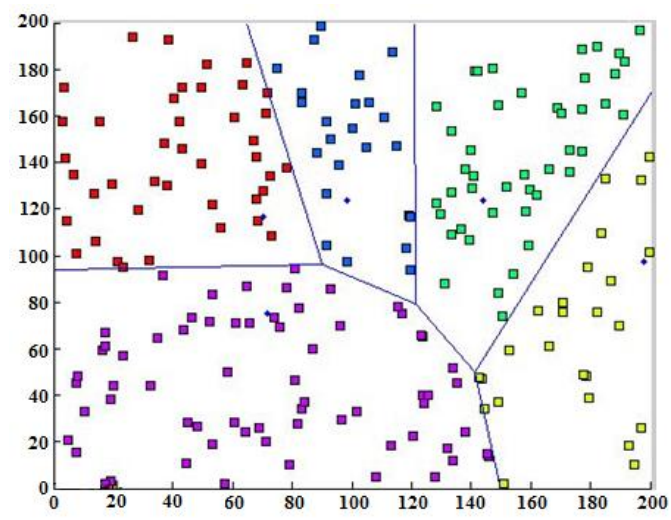

Figure 7. Network configuration for 200 nodes and 5 clusters and 5 cluster heads of proposed method in network size $200 \times 200$

As specified in table 2 and figure 8, the proposed method is better than IBLEACH and LEACH methods, taking into account the HND and FND criteria. Like in previous cases, LEACH has the lowest performance, because the reasons for low performance in the first case are true for this case. 
Table 2. FND and HND values of network size $200 \times 200$ in round 500

\begin{tabular}{|l|l|l|}
\hline Method & HND & FND \\
\hline LEACH & 850 & 425 \\
\hline IBLEACH & 860 & 595 \\
\hline Proposed & 1050 & 583 \\
\hline
\end{tabular}

The results of this situation clearly show that unequal clustering algorithms such as IBLEACH and the proposed method where to use a multi-hop routing protocol, have a better result. Because the batteries of the sensor nodes that are closer to the base station are discharged faster. However, these methods control this condition by decreasing the size of clusters near the base station. In this case, the IBLEACH performance in FND is higher than the three clustering. At LEACH, sensor nodes start to die sooner than the rest of the algorithms. At this criterion, the proposed method is $37 \%$ better than LEACH but $2 \%$ weaker than IBLEACH, which FND criterion is less important than the HND criterion.

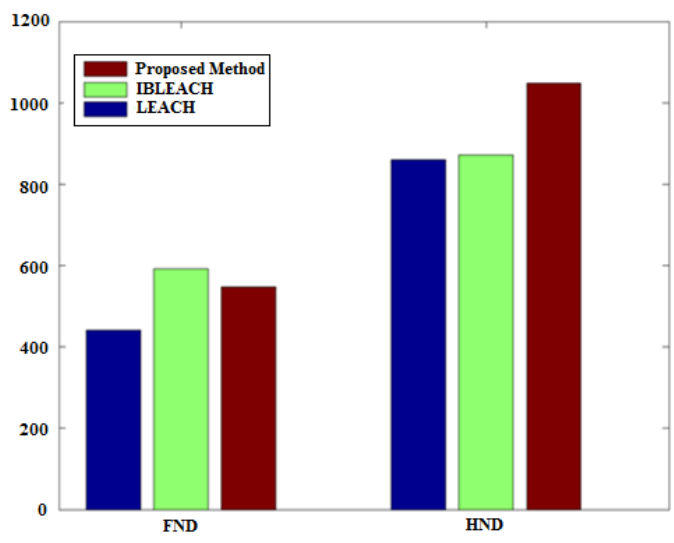

Figure 8. Death charts of the first node and half of nodes at 200 nodes in round 500

IBLEACH and proposed clustering algorithms with respect to the HND criterion have the better results than LEACH algorithm. If HND are criteria taken into account, the proposed method has better performance than IBLEACH and LEACH algorithms at sensor networks. In addition, the performance of LEACH in HND is significant, but still has less performance than the proposed method. At this criterion, the proposed method is $23 \%$ better than LEACH and $22 \%$ better than IBLEACH.

The total energy remaining in each algorithm for network size $200 \times 200$ and 2000 round is shown in figure 9. At Round 2000, LEACH has the lowest energy level of about 12 Jules. The energy level of IBLEACH is approximately 75 Jules. On the other hand, the proposed method has the highest energy level of 142 Jules. This result is concluded alongside the FND and HND criteria which is $1083 \%$ higher than LEACH and $89 \%$ higher than IBLEACH. 


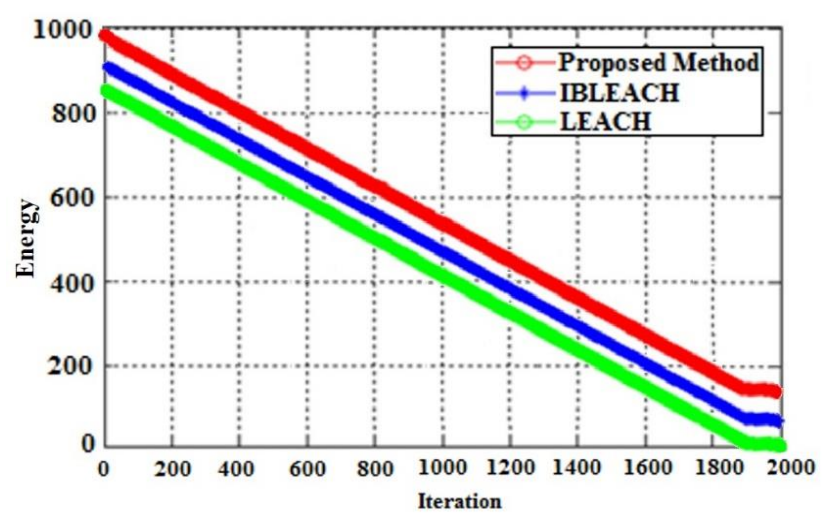

Figure 9. Remaining energy at 200 nodes in 2000 rounds

Figure 10 shows the number of live nodes according to the number of rounds in each algorithm. This figure clearly shows that the proposed algorithm tries to maintain nodes so that most of them survive simultaneously.

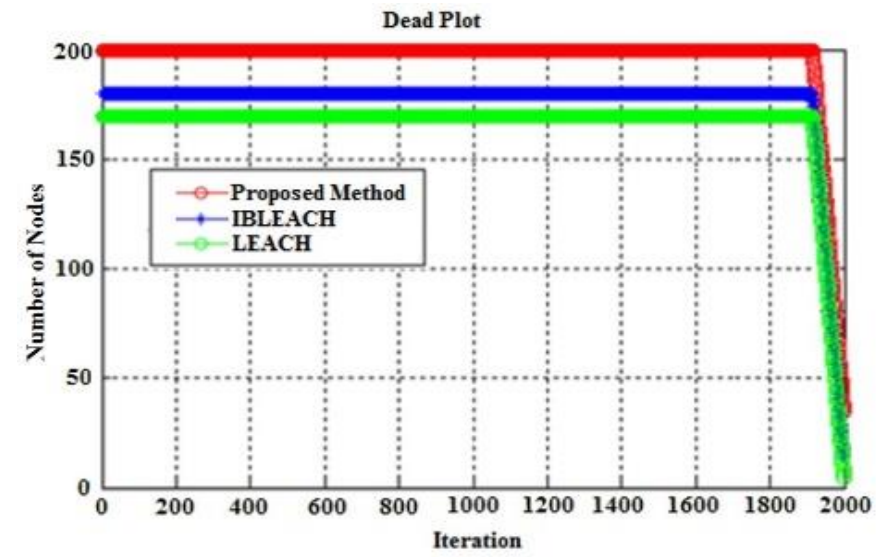

Figure 10. The number of live network nodes at 200 nodes in 2000 rounds

The number of cluster heads in different methods in different rounds for 200 nodes is shown in figure 11. Cluster heads in the proposed method are found in less rounds than two other methods, which indicates that the operation speed of the proposed method is better than the other two methods.

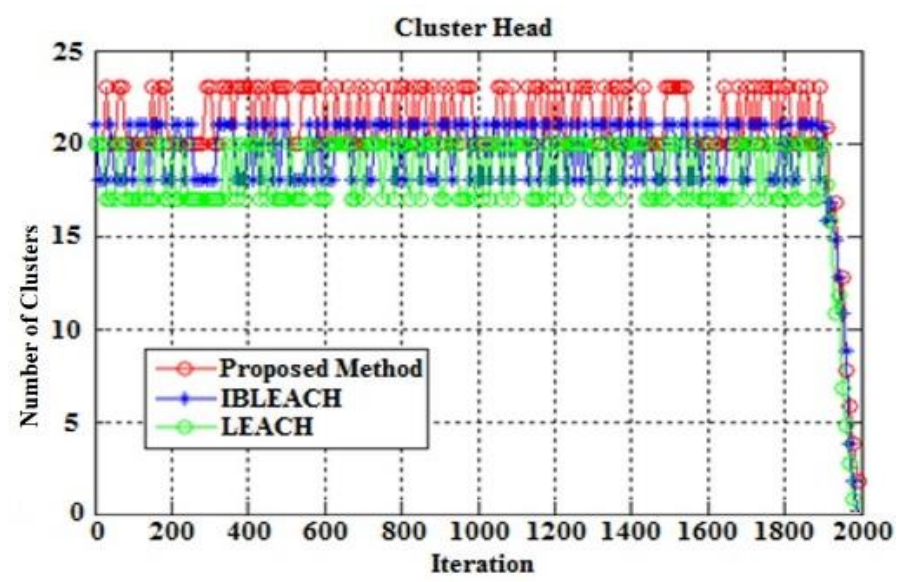

Figure 11. Number of cluster heads at 200 nodes in network size $200 \times 200$ and 2000 rounds 


\subsubsection{Results for 300 nodes}

The network configuration for 300 nodes with network size $300 \times 300$ and 5 initial clusters of proposed algorithm is shown in figure 12. Once the network is deployed, the simulation of this case leads to the results of table 3 .

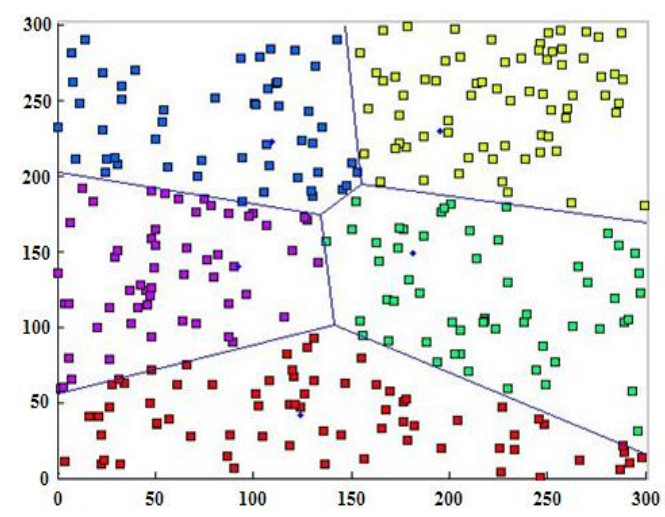

Figure 12. Network configuration for 300 nodes with 5 clusters of network size $300 \times 300$

As shown in table 3 and figure 13, the values of the HND and FND criteria for each algorithm have been reduced according to the preceding scenarios, due to the fact that the base station is outside the WSN. Therefore, the cluster head consumes more energy in transmitting its data packets to the base station.

Table 3. FND and HND values and network residual energy in round 500 at network size $300 \times 300$

\begin{tabular}{|l|l|l|}
\hline Method & HND & FND \\
\hline LEACH & 349 & 183 \\
\hline IBLEACH & 492 & 309 \\
\hline Proposed & 501 & 453 \\
\hline
\end{tabular}

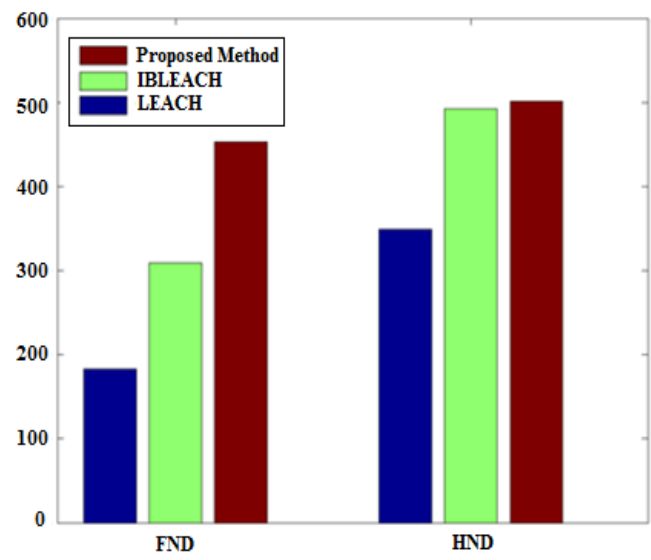

Figure 13. Death charts of the first node and half of nodes at 300 nodes in round 500

In this case, the proposed method in the both HND and FND criteria has better performance than LEACH and IBLEACH, while LEACH has the least performance in both of these criteria. In this case, the proposed clustering algorithms and IBLEACH in the FND criterion have a better 
performance than LEACH. This means that if the assignment of the values to the cluster head radius closer to the base station is smaller, the death of the sensor nodes can be delayed. The result of simulation shows that the proposed clustering approach is better, even if the base station is outside the network.

If the FND criterion is considered, the proposed method is better than LEACH with $147.5 \%$ and approximately $46.6 \%$ better than IBLEACH. If the HND criterion is considered, the proposed method is about $43.5 \%$ higher than LEACH and approximately $2 \%$ higher than IBLEACH.

Figure 14 shows the distribution of live sensor nodes according to the number of rounds in each simulated algorithm. Like the previous scenarios, the proposed algorithm attempts to keep all sensor nodes live simultaneously. Figure 15 shows the energy diagram of each algorithm according to the number of nodes, which is higher than other algorithms at any time. According to this figure, the proposed algorithm at round 3000 is $1400 \%$ better than LEACH and $97 \%$ better than IBLEACH in round 3000.

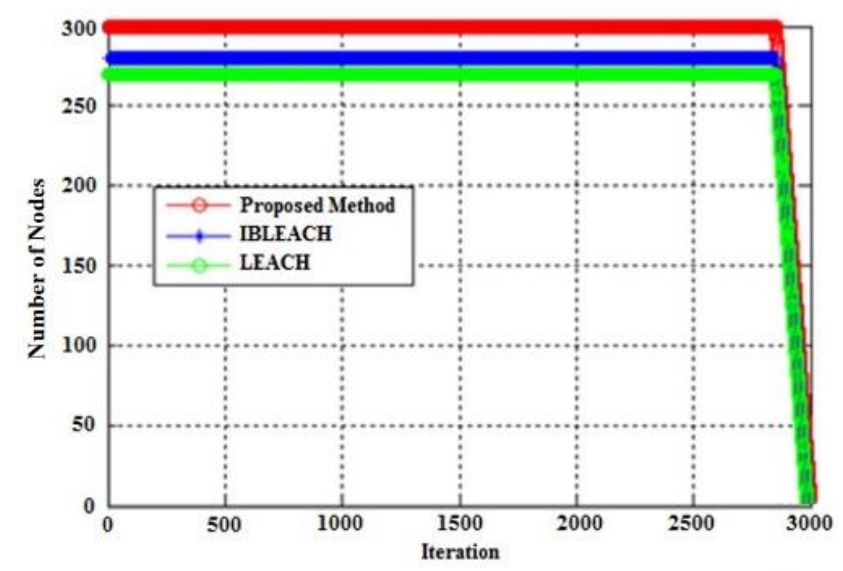

Figure 14. The number of live nodes in the network at 300 nodes in 3000 rounds

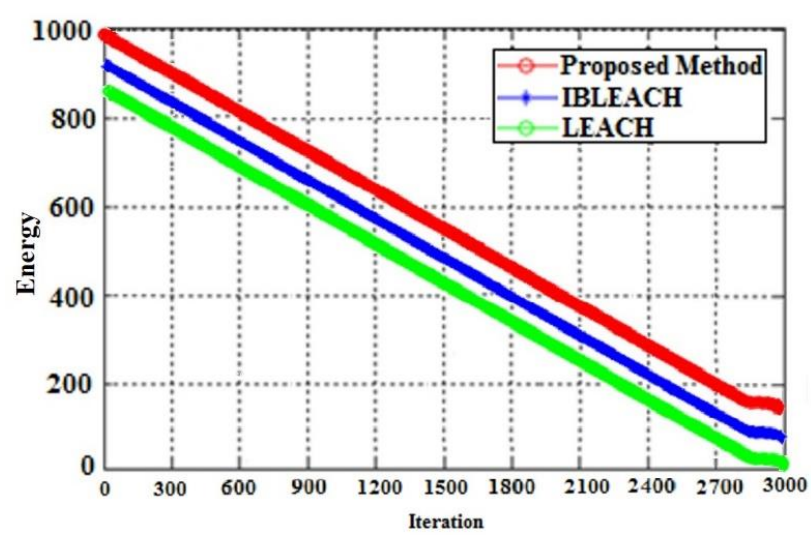

Figure 15. Remaining energy at 300 nodes in 3000 rounds

In figure 16, the diagram shows the number of cluster heads at 300 nodes. Head of clusters in the proposed method is indicated in fewer periods than two other methods, which indicates that the operation speed of the proposed method is better than the other two methods. 


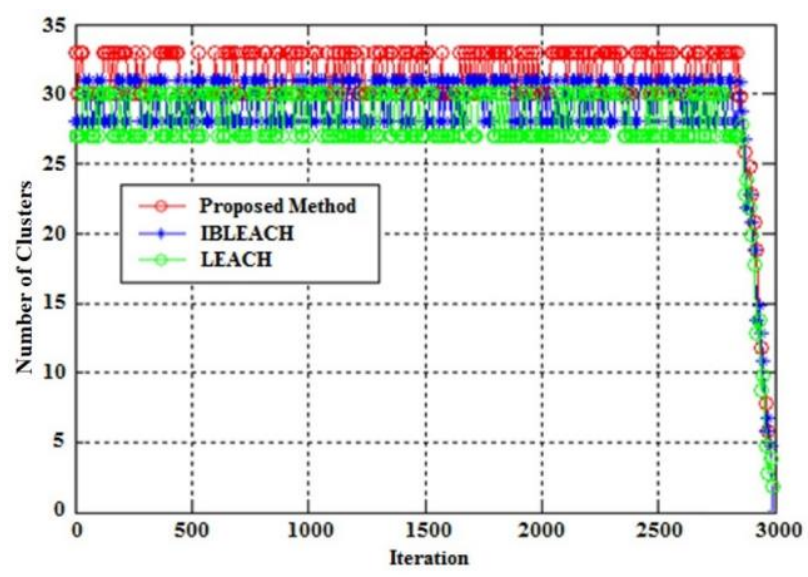

Figure 16. The number of cluster heads at 300 nodes in 3000 rounds

The more cluster heads in the first round, the better clustering will carry out. Since the proposed method has the largest number of clusters in the first round, therefore it naturally works better than two other methods.

\section{Conclusions}

In this paper, we outlined the results of the evaluation of the proposed method to reduce energy consumption in wireless sensor networks and compared the results of our method with LEACH and IBLEACH algorithms. According to the results of the evaluations, the proposed method has a higher efficiency than these two methods and delayed the death of the first node and half of the nodes in comparison to them.

In this research, a cluster-based solution was proposed to solve energy consumption problems in the sensor network. The performance evaluation of the proposed method showed that the proposed method greatly reduces energy consumption which is on average $40 \%$ better than LEACH algorithm and 14\% better than IBLEACH algorithm.

\section{REFERENCES}

[1] Eltaliawy, A., Mostafa, H. \& Ismail, Y., (2015) "Micro-scale variation-tolerant exponential tracking energy harvesting system for wireless sensor networks", Microelectronics Journal, Vol. 46, No. 3, pp221-230.

[2] Warneke, B.A. \& Pister K.S.J., (2002) "MEMS for distributed wireless sensor networks", 9th International Conference on Electronics, Circuits and Systems, 15-18 September, Dubrovnik, Croatia.

[3] Di Martino, G., Iodice, A., Riccio, D. \& Ruello, G., (2014) “A radio-frequency tool for planning wireless sensor networks”, Euro Med Telco Conference (EMTC), 12-15 November, Naples, Italy.

[4] Sun, Z. J., Li, W. B., Xiao, H. F. \& Xu, L., (2010) “The Research on Solar Power System of Wireless Sensor Network Node for Forest Monitoring”, International Conference on Web Information Systems and Mining, 23-24 October, Sanya, China.

[5] Al-Dahoud, A., Fezari, M. \& Belhouchet, F. Z., (2014) "Remote Monitoring System using WSN for Solar Power Panels ", First International Conference on Systems Informatics, Modelling and Simulation, 29 April-01 May, Sheffield, UK. 
International Journal of Computer Networks \& Communications (IJCNC) Vol.11, No.2, March 2019

[6] Gungor, V. V. C., Lu, B. \& Hancke, G. P., (2010) "Opportunities and challenges of wireless sensor networks in smart grid,” IEEE Transaction on Industrial Electronics, Vol. 57, No. 10, pp3557-3564.

[7] Borges, L. M., Velez, F. J. \& Lebres, A. S., (2014) "Survey on the Characterization and Classification of Wireless Sensor Network Applications", IEEE Communications Surveys \& Tutorials, Vol. 16, Issue 4.

[8] Sedighimanesh, M., Baqeri, J. \& Sedighimanesh, A., (2016) "Increasing Wireless Sensor Networks Lifetime with New Method", International Journal of Wireless \& Mobile Networks (IJWMN), Vol. 8, No. 4, pp65-80.

[9] Steenkamp, Leon du T., Kaplan, Shaun \& Wilkinson, Richardt H., (2009) "Wireless sensor network gateway", IEEE AFRICON, 23-25 September, Nairobi, Kenya.

[10] Peng, S., Wang, T. \& Low, C. P., (2015) "Energy neutral clustering for energy harvesting wireless sensors networks", Ad Hoc Networks, Vol. 28, No. 0, pp1-16.

[11] Heinzelman, Wendi Rabiner, Chandrakasan, Anantha \& Hari Balakrishnan, (2000) "Energy-Efficient Communication Protocol for Wireless Microsensor Networks", 33rd Annual Hawaii International Conference on System Sciences, 4-7 January, Maui, Hawaii, USA.

[12] Salim, A., Osamy, W. \& Khedr A. M., (2014) "IBLEACH: intra-balanced LEACH protocol for wireless sensor networks", Wireless Networks, Vol. 20, No. 6, pp1515-1525.

[13] Kuila, P. \& Jana, P.K., (2012) "Energy Efficient Load-Balanced Clustering Algorithm for Wireless Sensor Networks", Procedia Technology, Vol. 6, No. 0, pp771-777.

[14] Bandyopadhyay, S. \& Coyle, E.J., (2003) “An energy efficient hierarchical clustering algorithm for wireless sensor networks", 22th Annual Joint Conference of the IEEE Computer and Communications Societies (IEEE Cat. No.03CH37428), 30 March-3 April, San Francisco, CA, USA.

[15] Montiel, E. R., Rivero-Angeles, M. E., Rubino, G., Molina-Lozano, H., Menchaca-Mendez, R. \& Menchaca-Mendez, R., (2017) "Performance Analysis of Cluster Formation in Wireless Sensor Networks", Sensors, Vol. 17, No. 12, pp1-33.

[16] Bhanu, P. Uthaya \& Saravanan, J., (2014) "Data Security in Wireless Sensor Network", International Journal of Innovative Research in Computer and Communication Engineering, Vol. 2, Issue 2, pp3196-3204.

[17] Yadav, L. \& Sunitha, Ch., (2014) "Low Energy Adaptive Clustering Hierarchy in Wireless Sensor Network (LEACH)", International Journal of Computer Science and Information Technologies (IJCSIT), Vol. 5, No. 3, pp4661-4664.

[18] Bsoul, M., Al-Khasawneh, A., Abdallah, A. E., Abdallah, E. E. \& Obeidat, I., (2013) "An energyefficient threshold-based clustering protocol for wireless sensor networks", Wireless Personal Communications, Vol. 70, Issue 1, pp99-112.

[19] Ray, A. \& De, D., "Energy efficient cluster head selection in wireless sensor network", 1st International Conference on Recent Advances in Information Technology (RAIT), 15-17 March, Dhanbad, India.

[20] Chalak, A. R., Misra, S. \& Obaidat, M. S., (2010) "A cluster-head selection algorithm for Wireless Sensor Networks", 17th IEEE International Conference on Electronics, Circuits and Systems, 12-15 December, Athens, Greece.

[21] Azad, P. \& Sharma, V., (2013) "Cluster Head Selection in Wireless Sensor Networks under Fuzzy Environment”, ISRN Sensor Networks, Vol. 2013, pp1-8. 
[22] Tomar, G. S. \& Verma, S., (2009) "Dynamic Multi-Level Hierarchal Clustering Approach for Wireless Sensor Networks", 11th International Conference on Computer Modelling and Simulation, Cambridge, UK, 25-27 March.

[23] Attea, B. A. \& Khalil, E. A., (2011) “A new evolutionary based routing protocol for clustered heterogeneous wireless sensor networks”, Applied Soft Computing, Vol. 12, No. 7, pp1950-1957.

[24] MacQueen, J. B., (1967) "Some Methods for classification and Analysis of Multivariate Observations", 5th Berkeley Symposium on Mathematical Statistics and Probability, Berkeley, University of California Press, Vol. 1, pp281-297.

\section{AUTHOR}

Gholamreza Farahani received his BSc degree in electrical engineering from Sharif University of Technology, Tehran, Iran, in 1998 and MSc and PhD degrees in electrical engineering from Amirkabir University of Technology (Polytechnic), Tehran, Iran in 2000 and 2006 respectively. Currently, he is an assistant professor in the Institute of Electrical and Information Technology, Iranian Research Organization for Science and Technology (IROST), Iran. His research interest is computer networks especially routing.

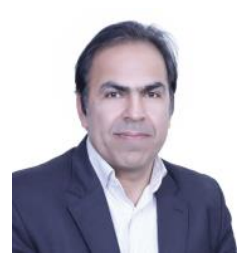

